\title{
Frequency Selection of Wake Flow Behind a NACA 0012 Wing
}

Han-Wen Lee

Professor, Department of Mechanical Engineering, National Taiwan University of Science and Technology, Taipei, Taiwan, R.O.C., rfhuang@mail.ntust.edu.tw

Rong-Fung Huang

Graduate student, Department of Mechanical Engineering, National Taiwan University of Science and Technology, Taipei, Taiwan, R.O.C.

Follow this and additional works at: https://jmstt.ntou.edu.tw/journal

Part of the Mechanical Engineering Commons

\section{Recommended Citation}

Lee, Han-Wen and Huang, Rong-Fung (1998) "Frequency Selection of Wake Flow Behind a NACA 0012 Wing," Journal of Marine Science and Technology. Vol. 6: Iss. 1, Article 4.

DOI: $10.51400 / 2709-6998.2517$

Available at: https://jmstt.ntou.edu.tw/journal/vol6/iss1/4

This Research Article is brought to you for free and open access by Journal of Marine Science and Technology. It has been accepted for inclusion in Journal of Marine Science and Technology by an authorized editor of Journal of Marine Science and Technology. 


\title{
FREQUENCY SELECTION OF WAKE FLOW BEHIND A NACA 0012 WING
}

\author{
Han-Wen Lee** and Rong-Fung Huang*
}

Keywords: Wake, Vortex shedding, Instability.

\begin{abstract}
The oscillating flow modes, frequency selection, and response of periodic frequency to change of characteristic flow modes behind a NACA 0012 wing model are studied experimentally. The functional relationships of the dominant mechanisms in limiting ranges of Reynolds number are justified analytically. The oscillating instabilities in wake region possess four characteristic modes: laminar, subcritical, transitional, and supercritical. In viscous effect dominated regime of laminar mode, Strouhal number is inversely proportional to Reynolds number. In inertial effect or pressure gradient dominated regime of supercritical mode, Strouhal number remains constant. In regime between limiting cases, Strouhal number increases nonlinearly with Reynolds number and drops to a low value as shed vortices change from laminar to subcritical mode. The increase of free-stream turbulent fluctuations causes the decrease of Strouhal number only at low Reynolds numbers. In high Reynolds number regime, the effect is not apparent.
\end{abstract}

\section{INTRODUCTION}

There were many studies over the years on the nature of flow separation and wake of airfoil operating at low Reynolds numbers in an effort to solve many significant steady/unsteady aerodynamic problems occurring at chord Reynolds numbers lower than $5 \times 10^{5}$. Most of the studies were focused on the behavior and control of the boundary-layer on the suction surface, e.g., laminar separation, transition, turbulent reattachment, bubble bursting, etc., due to its significant influences on the aerodynamic performance. Lissaman [1] and Gad-el-Hak [2] have made excellent reviews on the characteristics and control of

Paper Received November, 1997. Revised March, 1998. Accepted April, 1998. Author for Correspondence: Rong Fung Hwang.

* Professor, Department of Mechanical Engineering, National Taiwan University of Science and Technology, Taipei, Taiwan, R. O.C. E_mail: rhuang@mail.ntust.edu.tw

**Graduate student, Department of Mechanical Engineering, National

Taiwan University of Science and Technology, Taipei, Taiwan, R.O.C. the surface flows of the low-Reynolds number airfoils. Another important subject of research was the unsteady flow in the wake of the airfoil. The wake behind an airfoil at low Reynolds numbers usually consists of instability waves and coherent structures with periodic unsteady motions.

The unsteady flow behind the airfoil has profound influences on the airfoil performance. Of the most interesting behaviors related to the periodic unsteady flow are the vortex-induced vibration $[3,4]$ of the airfoil and the lock-on phenomenon [5] of the oscillating frequency of vortex shedding. Traditionally, the researches of the oscillating flows were focused on the basic flows, such as the wake behind a bluff body [6-11], the shear layer in the jet or mixing layer $[12,13]$, or the wake behind the slender body with blunt trailing edge $[14,15]$. However, the literature on the detailed behaviors, dominant mechanisms, and frequency selection of the unsteady wake behind the airfoil was limited. The unsteady wake of the airfoil at high angles of attack and large Reynolds numbers was found to behave similarly to that of the bluff body [16]. The frequency selection of the vortex shedding behind a bluff body has been widely studied. Most wake oscillations behind the bluff body reported in the literature were commonly translated to an "ordinary" Strouhal number $S t=f d / u$, where $f$ is the flow oscillation frequency, $d$ the cross-stream length scale of the body, and u the free-stream velocity. Roshko [17] found that the ordinary Strouhal number remains near constant $0.21,0.18$, and 0.14 for circular cylinder, $90^{\circ}$ wedge, and bluff plate, respectively, for the Reynolds number in the range between $10^{3}$ and $10^{5}$. Simons [18] further introduced an "universal" Strouhal number $S t^{*}$ based on the measured wake width $d^{*}$ for the two-dimensional bluff bodies and found a constant value of $S t^{*}=0.163$. Roshko [17] also applied successfully the inviscid Kirchhoff's free streamline theory to relate the drag and the universal Strouhal number by a $k$ function, which implied the appropriation of the inviscid model in the bluff-body wake at large Reynolds numbers. Levi [10] justified 
the universal Strouhal law by modeling the available specific kinetic energy, $u^{2} / 2$, of the oscillating fluids by the specific mechanical energy, $\left(2 \pi f d^{*}\right)^{2} / 2$, of the oscillator oscillating within the width $d^{*}$ with a frequency $f$. The universal Strouhal number $S t^{*}=1 / 2 \pi$ $=0.159$ was derived. The Karman type vortex street behind an airfoil was discussed by Stuber and Gharib [19]. Zaman et al. [20] reported that during deep stall of a LRN(1)-1007 airfoil at angles of attack larger than $18^{\circ}$, the usual "bluff-body" shedding occurs at the ordinary Strouhal frequency 0.2 in the chord Reynolds number range of $0.15 \times 10^{5}-3.0 \times 10^{5}$. But at the onset of static stall around angle of attack $15^{\circ}$, a low-frequency periodic oscillation with a Strouhal number about one order of magnitude smaller than the Strouhal frequency was observed. Huang and Lin [21] presented that for a NACA0012 wing at angles of attack larger than $15^{\circ}$, the diverse distribution of the ordinary Strouhal numbers collapses gradually to a narrow band at chord Reynolds numbers between $0.11 \times 10^{5}-1.0 \times 10^{5}$ and approaches to a value 0.12 at $90^{\circ}$ angle of attack. The inviscid behavior in the unsteady wake of the airfoil at high angles of attack and large Reynolds numbers is anticipated.

For the airfoils with sharp trailing edge at low angles of attack without separation of surface flow, the wake with the characteristics of mixing-layer developing spatially from the instability waves of small amplitude to roll-up vortices was observed [21]. As the viscous force is not negligible in analyzing the evolving process of the instability waves at the initial stage of the spatially developing vortex street in a free shear layer [22], the frequency selection of the mixing layer is different from that of the bluffbody wake. Although the classical inviscid stability theorem was able to predict an universal Strouhal number 0.032 in the initial region of instability waves in a perturbed free shear layer [12], the unsteady wake behind the airfoil with separation of surface flow seems not to fall completely in the category. Nishika and Sato [23] showed that the shedding frequency behind a circular cylinder at very low Reynolds numbers is determined by the selective linear growth of small fluctuations. At higher Reynolds numbers, however, nonlinear effects dominate. The dominant mechanisms of the unsteady flow in the wake of an airfoil at low angles of attack and Reynolds numbers are inevitably different from those at high angles of attack and Reynolds numbers. Several characteristic modes of the oscillating unsteady flow were identified and found to be closely related to the boundarylayer behavior on the airfoil surfaces [21]. However, the frequency selection, response to the change of shedding mode, as well as the functional relationship

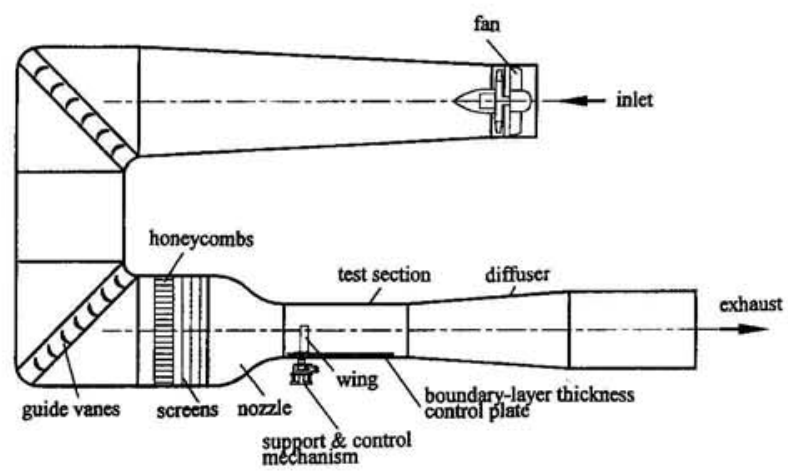

Fig. 1. Experimental set-up.

of the dominant mechanisms in different ranges of Reynolds number and angle of attack were not reported in the literature.

Although the flow characteristics of the lowReynolds number wings have been comprehensively studied, a systematic survey of the unsteady flow in the Reynolds number/angle of attack domain is still an open area for the researchers. In this paper, the experimental results of the frequency selection and characteristic modes of the vortex shedding behind a NACA0012 wing are presented. A theoretical analysis using the simplified Navier-Stokes equations in limiting situations based on the similarity theory and dimensional analysis is conducted to assist the interpretation of the experimental results and the dominating mechanisms. The influences of the freestream turbulence intensity on the vortex shedding characteristics are also discussed.

\section{EXPERIMENTAL EQUIPMENT}

The experiments were conducted in an open loop, low speed wind tunnel with an anti-turbulence honeycomb section, three wire-mesh screens, a 10:1 contractor, and a test section of $60 \times 60 \times 120 \mathrm{~cm}$, as shown in Fig. 1. The free-stream turbulence intensity is less than $0.2 \%$ within the operating range. During the experiments the velocity of the approaching flow was monitored with a Pitot-static tube together with a calibrated Validyne pressure transducer. An aluminum plate with sharp leading and trailing edges is placed $5 \mathrm{~cm}$ above the floor of the test section for the control of the boundary-layer thickness. A straight wing model made of stainless steel with the NACA0012 profile [24] of cross section, $6 \mathrm{~cm}$ chord length, and $30 \mathrm{~cm}$ span is mounted on a support and protrudes through perpendicularly to the aluminum floor of the test section and the boundary-layer thickness control plate. The support for the wing model has a resolution of 0.015 degrees when doing revolu- 
tion. The accuracy of the angle of attack is controlled within $0.5 \%$.

The frequencies of the oscillating instabilities in the wake region are detected by a hot-wire anemometer. The dynamic response of the hot-wire anemometer to the testing square waves is regulated to about $20 \mathrm{kHz}$. The hot-wire signals are fed simultaneously to an FFT analyzer and a high-speed PC-based data acquisition system to perform analysis and calculations of dynamic behaviors and statistics. The accuracy of the shedding frequency depends not only on the response of the hot-wire anemometer but also on the record length and sampling rate of the FFT analyzer. The uncertainty of the frequency detected is estimated to be within 1.5 percent in this experiment.

\section{RESULTS AND DISCUSSION}

\section{Characteristic Modes}

The instabilities in the wake of the wing are detected by placing a hot-wire anemometer in the downstream area. Four characteristic instability modes: laminar, subcritical, transitional, and supercritical, are identified in the domain of the free stream velocity $u_{w}$ and angle of attack $\alpha$, as shown in Fig. 2(a). Figure 2(b) shows the mode identification in the domain of the Reynolds number $R_{e d}$ and angle of attack $\alpha$. The typical hot-wire signals of laminar mode shown in Fig. 3(a) are observed in the regime of low Red and $\alpha$ of Fig. 2(b). The signals are smooth and periodic. In the regime of the subcritical mode of Fig. 2(b), the periodic hot-wire signals superimposed by small fluctuations appear, as shown in Fig. 3(b). The turbulent fluctuations develop with the evolution of shedding vortices. In the regime of transitional mode of Fig. 2(b), the hot-wire signals with random fluctuations appear, as shown in Fig. 3(c). No particular peak is found in the power spectrum of the velocity signals. The shed vortices lose coherency and the flow structure in the wake is disorganized, probably because of the mixing effect induced by the enhanced turbulence intensity. In the regime of supercritical mode of Fig. 2(b), the periodic hot-wire signals superimposed by large turbulent fluctuations are present, as shown in Fig. 3(d). The turbulent vortices are shed in the wake. The characteristic modes of the vortex shedding in the wake region are closely related to the behaviors of the boundary layer on the suction surface of the wing [22].

\section{Frequency Selection}

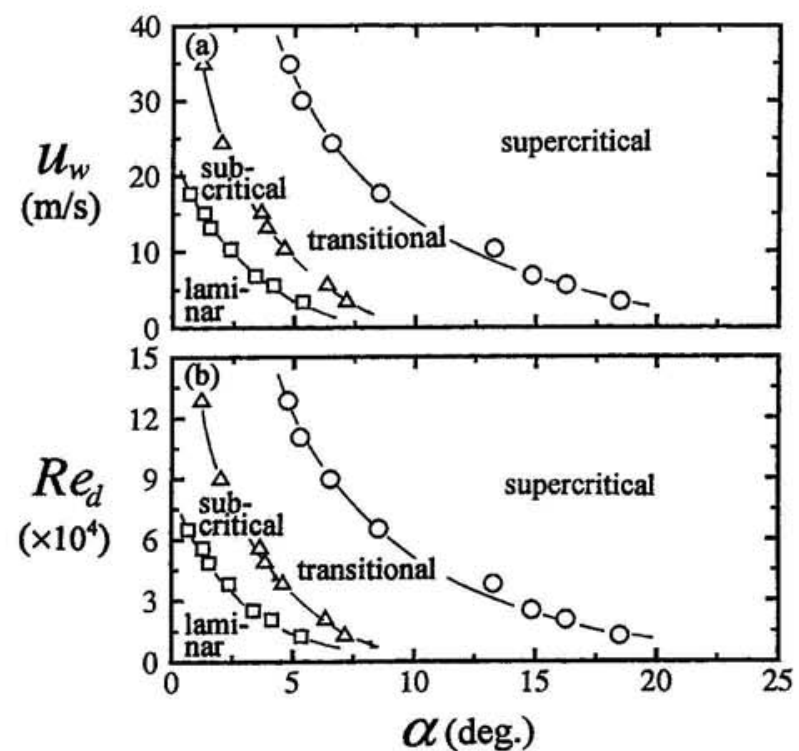

Fig. 2. Regimes of characteristic modes of wake flow. (a) in domain of free-stream velocity and angle of attack, (b) in domain of chord Reynolds number and angle of attack.

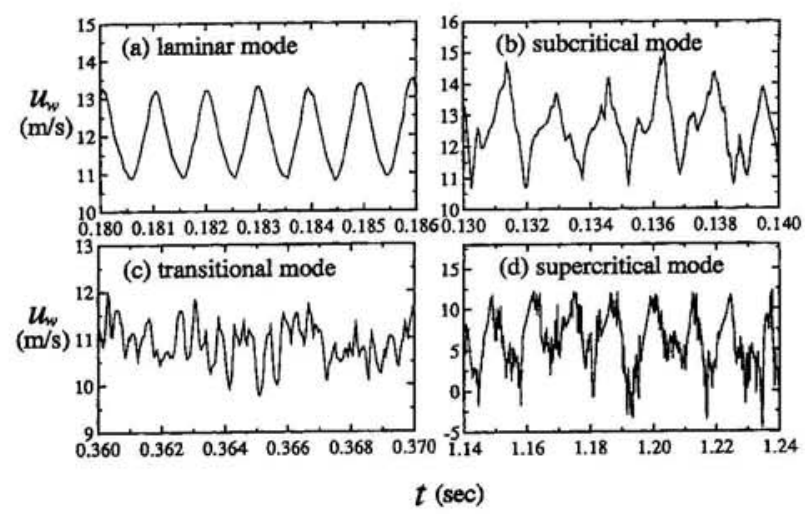

Fig. 3. Typical hot-wire signals of four characteristic modes.

The frequencies of the periodic unsteady motions in the wake varying with the wind velocity at low angles of attack are shown in Fig. 4(a). At $u_{w}$ lower than about $1 \mathrm{~m} / \mathrm{s}$, the frequencies at all angles of attack remain constant at about $15 \mathrm{~Hz}$. At $u_{w}$ larger than about $1 \mathrm{~m} / \mathrm{s}$, the frequencies increase nonlinearly with the increase of free stream velocity. As the unsteady motions change mode from laminar to subcritical regime, the frequencies drop sharply to small values, then increase with uw almost linearly. The increase rate of $f$ in subcritical regime is not as high as that in laminar regime. At low angles of attack, the frequencies in the laminar mode vary only a little with $\alpha$. However, the frequency differences are apparent in the subcritical regime between angles of attack. The data of Fig. 4(a) are re-plotted with non-dimen- 

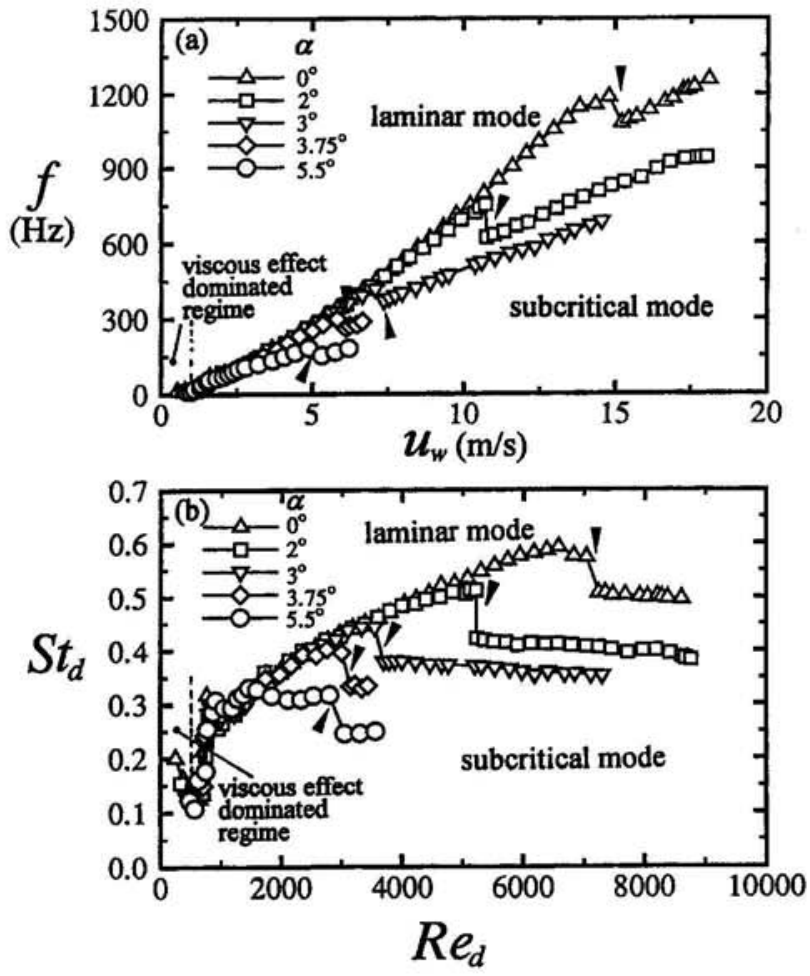

Fig. 4. Frequency selection of oscillating wake structures at low angles of attack. (a) frequency vs. free-stream velocity, (b) Strouhal number vs. Reynolds number.

sional groups $S t_{d}$ and $R e_{d}$, as shown in Fig. 4(b). In laminar regime, the Strouhal numbers at low angles of attack do not differ apparently. At Reynolds numbers lower than about 600 , the Strouhal number decreases from 0.2 to 0.1 with the increase of $R e_{d}$. At Red larger than about 600 , the Strouhal number increases with the increase of Reynolds number. The Strouhal numbers drop to low values as the periodic motions change mode from laminar to subcritical regime, then decrease slightly with the increase of $R e_{d}$. The increase of turbulent fluctuations superimposed on the periodic motions with the increase of Reynolds number leads to the slight decrease of $S t_{d}$ [12]. At large angles of attack, the vortices are shed in supercritical mode where the periodic motions are superimposed by large turbulent fluctuations. The frequencies of the periodic motions increase almost linearly with the increase of free stream velocity, as shown in Fig. 5(a). The shedding frequency decreases with the increase of angle of attack. Figure 5(b) shows the variations of Strouhal number with Reynolds number at various angles of attack. The Strouhal numbers in the supercritical mode remain constant with the increase of Reynolds number at fixed angle of attack. The value of Std decreases
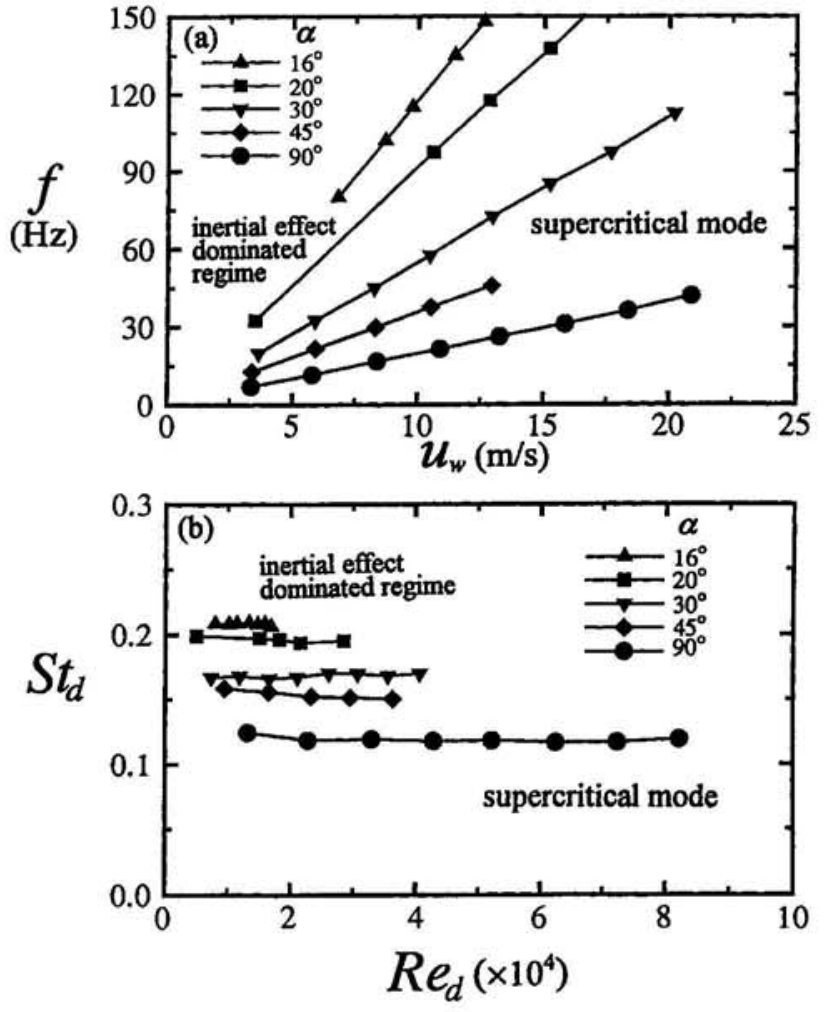

Fig. 5. Frequency selection of oscillating wake structures at high angles of attack. (a) frequency vs. free-stream velocity, (b) Strouhal number vs. Reynolds number.

from about 0.21 at $\alpha=16^{\circ}$ to 0.12 at $\alpha=90^{\circ}$.

\section{Influences of Free-Stream Turbulence}

The turbulence intensity of free stream is increased by setting a mesh screen $20 \mathrm{~cm}$ up stream of the wing model. With the increase of the turbulence intensity of free stream to $0.5 \%$, the boundaries identifying the characteristic modes change significantly, as shown in Fig. 6. The changes from laminar to subcritical mode as well as from subcritical to transitional mode occur at lower Reynolds numbers and angles of attack. The regime of subcritical mode becomes hardly distinguishable. In Fig. 7 the Strouhal number decreases firstly with the increase of Reynolds number at low $R e_{d}$, then increases at large Reynolds numbers. At Reynolds numbers lower than about 1500 , the values of $S t_{d}$ are lower than those in Fig. 4(b). The Strouhal numbers of vortex shedding of laminar mode at low angles of attack collapse to almost a single curve. At $\alpha=5.5^{\circ}$, the deviation is apparent. At Reynolds numbers larger than about 1500 , the values of Std are almost the same as those in Fig. 4(b) of low free-stream turbulence intensity. At high angles of attack and Reynolds numbers, as shown 


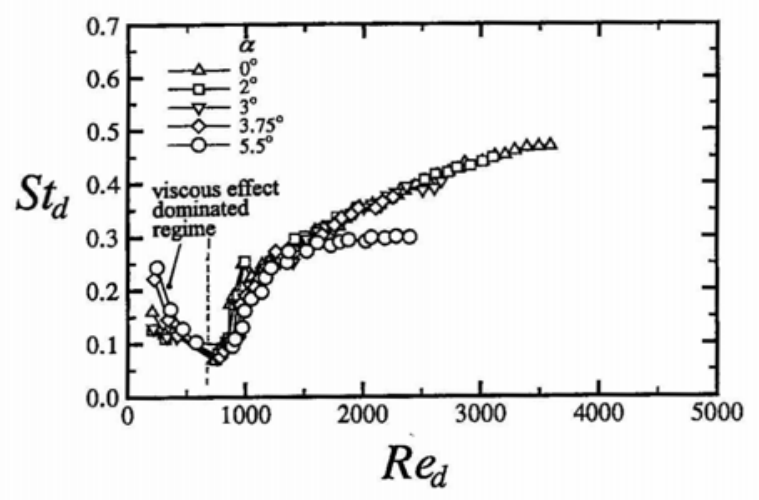

Fig. 6. Regimes of characteristic modes of wake flow. Free-stream turbulence intensity $0.5 \%$.

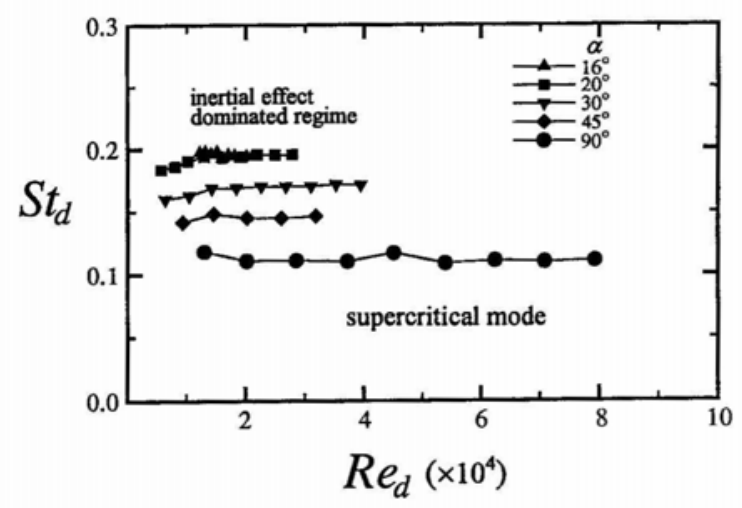

Fig. 7. Frequency selection of oscillating wake structures at low angles of attack. Free-stream turbulence intensity $0.5 \%$.

in Fig. 8, the free-stream turbulence intensity seems not to alter the values of Strouhal number when compared with those in Fig. 5(b). The free-stream turbulence intensity has an effect on the decreasing of the Strouhal number of the periodic motions only at low Reynolds numbers. At high Reynolds numbers, the effect is not apparent in the subcritical and supercritical regimes where the turbulent fluctuations superimpose on the periodic motions.

\section{Analytical Justification}

The measured results of the periodic frequencies in the wake behind the wing model show two asymptotic behaviors: the Strouhal numbers decrease at very low Reynolds numbers and remain constant in supercritical regime with the increase of $R e_{d}$. The asymptotic behaviors are justified by considering the limiting cases as follows.

Consider the incompressible, two-dimensional governing equations of continuity and momentum

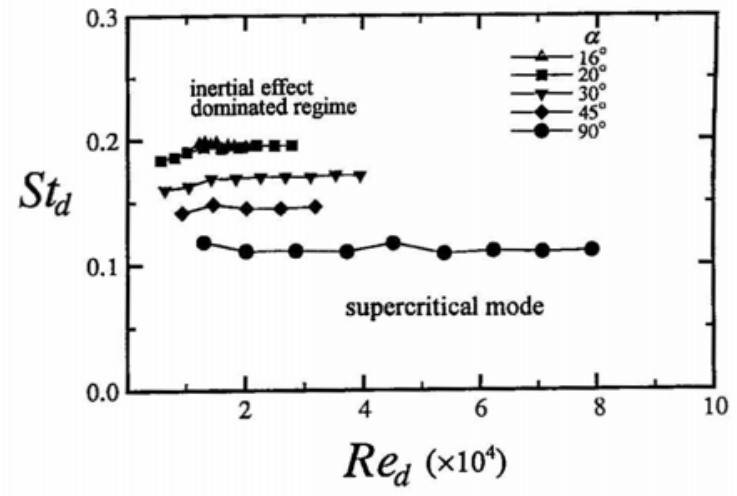

Fig. 8. Frequency selection of oscillating wake structures at high angles of attack. Free-stream turbulence intensity $0.5 \%$.

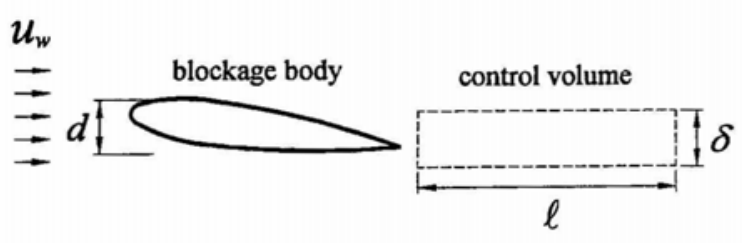

Fig. 9. Control volume for analytical analysis.

$$
\begin{aligned}
& \frac{\partial u}{\partial x}+\frac{\partial v}{\partial y}=0, \\
& \frac{\partial u}{\partial t}+u \frac{\partial u}{\partial x}+v \frac{\partial u}{\partial y}=-\frac{1}{\rho} \frac{\partial p}{\partial x}+v\left(\frac{\partial^{2} u}{\partial x^{2}}+\frac{\partial^{2} u}{\partial y^{2}}\right),
\end{aligned}
$$

and

$$
\frac{\partial v}{\partial t}+u \frac{\partial v}{\partial x}+v \frac{\partial v}{\partial y}=-\frac{1}{\rho} \frac{\partial p}{\partial y}+v\left(\frac{\partial^{2} v}{\partial x^{2}}+\frac{\partial^{2} v}{\partial y^{2}}\right)
$$

to the control volume placed in the wake region of the wing model, as shown in Fig. 9. Take $\ell$ and $\delta$ as the characteristic length scales in the $x$ and $y$ directions, respectively, so that the characteristic velocity scales uw and $V$ in $x$ and y directions, respectively, can satisfy the continuity equation. Let $T$ be the characteristic time scale of the instabilities. There hence is a corresponding characteristic quantity in frequency domain, $f=1 / T$. Balance of the order of magnitude of the terms of the continuity equation gives

$$
\frac{\partial u}{\partial x} \approx \frac{\partial v}{\partial y}
$$

With the help of the similarity theory [25], the characteristic quantities can thus be related by the following equation

$$
\frac{u_{w}}{\ell} \propto \frac{V}{\delta},
$$


in which the symbol " $\propto$ " denotes "proportional to". Since it is impractical to solve analytically the whole momentum equations (2) and (3), three limiting cases: viscous, inertial, and pressure effect dominated regimes, are considered separately for the instabilities in the wake at this stage.

In the case where the viscous effect dominates the unsteady motions, equations (2) and (3) are simplified to

$$
\frac{\partial u}{\partial t} \approx v\left(\frac{\partial^{2} u}{\partial x^{2}}+\frac{\partial^{2} u}{\partial y^{2}}\right)
$$

and

$$
\frac{\partial v}{\partial t} \approx v\left(\frac{\partial^{2} v}{\partial x^{2}}+\frac{\partial^{2} v}{\partial y^{2}}\right)
$$

By equating the order of magnitude of the unsteady term and the individual diffusion terms in above equations and applying successively the similarity theory with the characteristic scales $U_{w}, V$, $\ell$, and $\delta$, the following results are obtained

$$
\begin{aligned}
& x \text { component: } \frac{f \ell^{2}}{v} \propto \text { constant, } \\
& y \text { component: } \frac{f \delta^{2}}{v} \propto \text { constant. }
\end{aligned}
$$

Equation (8) and (9) can further be written as the product of Strouhal and Reynolds numbers:

$$
S t_{\ell} \cdot \operatorname{Re}_{\ell} \approx \text { constant, }
$$

and

$$
S t_{\delta} \cdot \operatorname{Re}_{\delta} \approx \text { constant. }
$$

The length scales $\ell$ and $\delta$ are usually not convenient for applications because they require exhausting measurements. Since $\ell$ and $\delta$ are functions of the cross-stream dimension of the obstacle at given free stream velocity [16-19], in most applications [16,17] they are lumped to the single physical variable $d$ so that the equations (8) to (11) are written as

$$
\frac{f d^{2}}{v} \propto \text { constant, }
$$

and

$$
S t_{d} \cdot R e_{d} \approx \text { constant. }
$$

Thus, in the regime where the viscous effect dominates the unsteady motions, the Strouhal number is approximately inversely proportional to the Reynolds number. The results in Fig. 4(b) at Reynolds numbers lower than about 600 show the characteris-

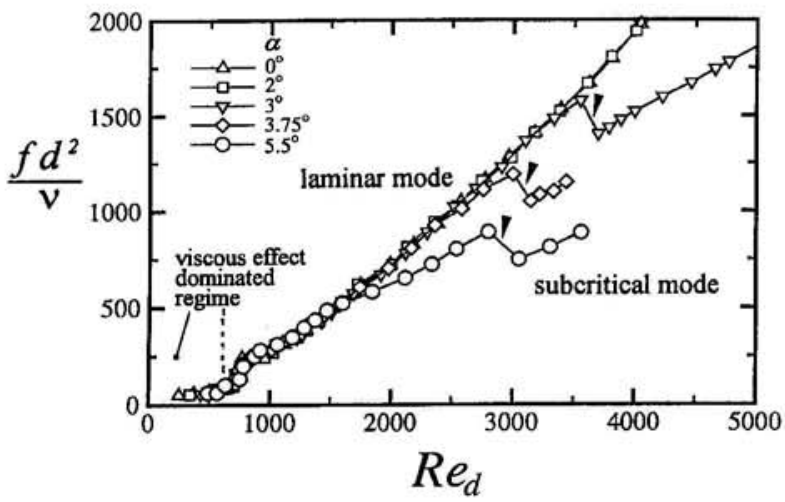

Fig. 10. Frequency selection of oscillating wake structures in terms of Roshko number at low angles of attack.

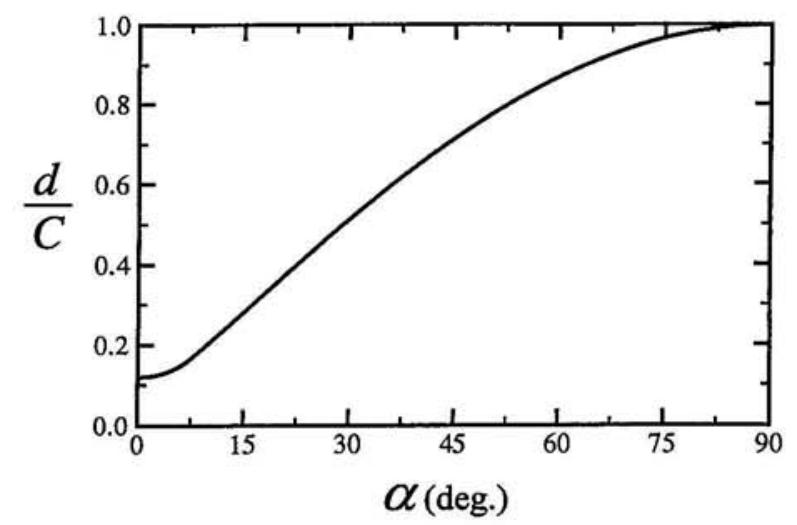

Fig. 11. Cross-stream projection of wing section.

tics of viscous effect dominated regime. The nondimensional group $\frac{f d^{2}}{v}$ in equation (12) was conven tionally called the Roshko number and was adopted by many investigators to correlate the periodic wakes [6-8]. In Fig. 10, the Roshko number is plotted via the Reynolds number. The values of $\frac{f d^{2}}{v}$ remain constant about 60 at Reynolds numbers lower than 600 . Since the values of $\mathrm{d}$ for NACA 0012 wing do not change much at angles of attack lower than $4^{\circ}$, as shown in Fig. 11, and $v$ of air stream is a constant, the values of $f$ at low angles of attack do not vary significantly in the viscous effect dominated regime of Fig. 4(a).

In the case of the inertial effect dominated regime, follow similar procedure of obtaining equations (8)-(11), apply the balance of order of magnitude, similarity theory, and equation (5), and take the physical cross stream dimension $d$ to replace the length scale $\ell$, the momentum equations are reduced to

$$
\frac{f d}{u_{w}} \propto \text { constant, }
$$




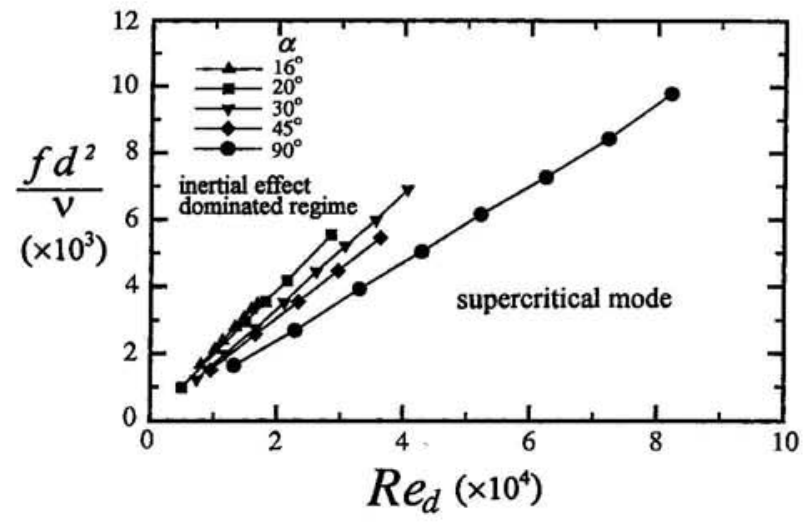

Fig. 12. Frequency selection of oscillating wake structures in terms of Roshko number at high angles of attack.

or

$$
S t_{d} \propto \text { constant. }
$$

The Strouhal numbers which remain constant at high Reynolds numbers are observed in Fig. 7(b). The equation (14) can be further written as $\frac{f d^{2}}{v} \propto \frac{u_{w} d}{v}$ so that

$$
\frac{f d^{2}}{v} \propto \operatorname{Re}_{d}
$$

Figure 12 shows the values of $\frac{f d^{2}}{v}$ at various angles of attack which are proportional to $R e_{d}$.

Consider the case where the pressure gradient dominates the unsteady motions. Let $\bar{x} \equiv x / \ell$, $\bar{y} \equiv y / \delta, \bar{u} \equiv u / u_{w}, \quad \bar{v} \equiv v / V$, and $\bar{t} \equiv t / T$, then equations (1)-(3) can be put in the non-dimensional forms

$$
\begin{aligned}
& \frac{\partial \bar{u}}{\partial \bar{x}}+\frac{\partial \bar{v}}{\partial \bar{y}}=0, \\
& S t_{\ell} \frac{\partial \bar{u}}{\partial \bar{t}}+\bar{u} \frac{\partial \bar{u}}{\partial \bar{x}}+v \frac{\partial \bar{u}}{\partial \bar{y}} \\
& =-\frac{\partial \bar{p}}{\partial \dot{x}}+\frac{1}{\operatorname{Re}_{\ell}}\left[\frac{\partial^{2} \bar{u}}{\partial \bar{x}^{2}}+\left(\frac{\ell}{\delta}\right)^{2} \frac{\partial^{2} \bar{u}}{\partial \bar{y}^{2}}\right],
\end{aligned}
$$

and

$$
\begin{aligned}
& S t_{\ell} \frac{\partial \bar{v}}{\partial \bar{t}}+\bar{u} \frac{\partial \bar{v}}{\partial \bar{x}}+\bar{v} \frac{\partial \bar{v}}{\partial \bar{y}} \\
& =-\left(\frac{\ell}{\delta}\right)^{2} \frac{\partial \bar{p}}{\partial \bar{y}}+\frac{1}{\operatorname{Re}_{\ell}}\left[\frac{\partial^{2} \bar{v}}{\partial \bar{x}^{2}}+\left(\frac{\ell}{\delta}\right)^{2} \frac{\partial^{2} \bar{v}}{\partial \bar{y}^{2}}\right],
\end{aligned}
$$

where $\bar{p} \equiv p / \rho u_{w}^{2}$. By observing equations (18) and (19), the quantities $\rho u_{w}^{2}$ and $\rho u_{w}^{2}\left(\frac{\ell}{\delta}\right)^{2}$ are chosen as the characteristic pressure scales in $x$ and $y$ directions, respectively. Consider the balance of order of magni-
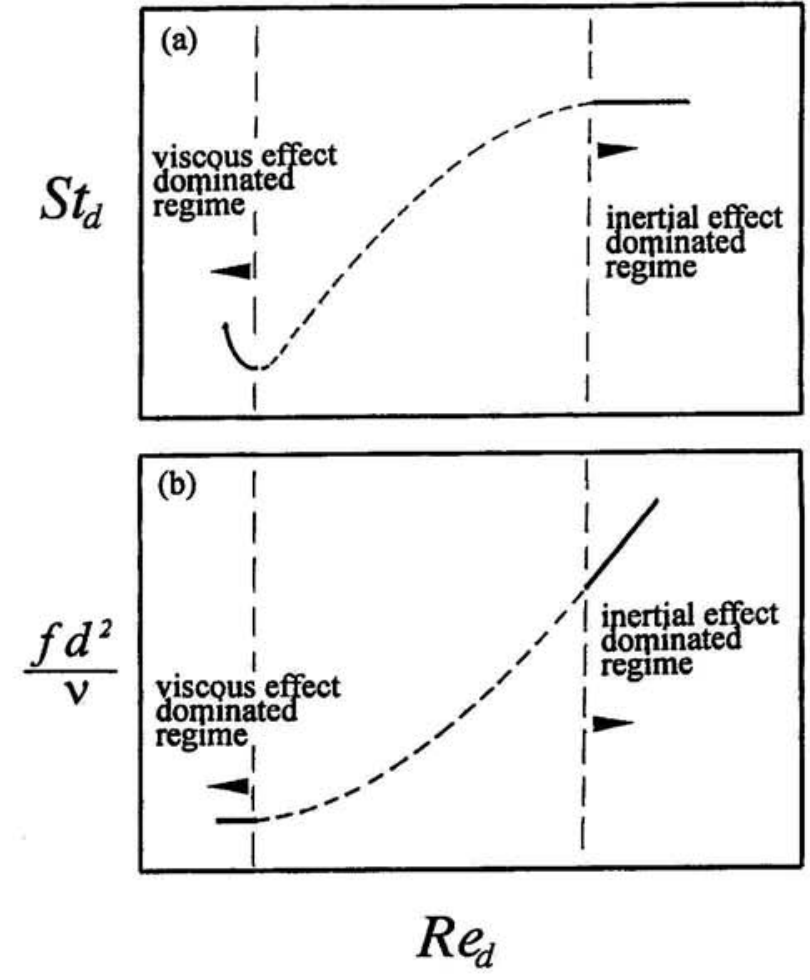

Fig.13. Schematic summary of instabilities of wake structures at limiting cases derived by analytical justification.

tude between the pressure and unsteady terms in equations (2) and (3), and apply the similarity theory by employing the appropriate characteristic scales, the same results as equations (14)-(16) are obtained.

The effect of the pressure gradients on the Strouhal number of unsteady motions is similar to that of inertial forces. The analytical results in the limiting cases are summarized schematically in Figs. 13(a) and (b) for $S t_{d}$ vs. $R e_{d}$ and $\frac{f d^{2}}{v}$ vs. $R e_{d}$, respectively. The general rules of these results are expected to be applied to unsteady motions in the wake region.

\section{CONCLUDING REMARKS}

The characteristics of the vortex shedding in the wake region behind the NACA0012 wing model are found to be multi-modes. Four characteristic modes - laminar, subcritical, transitional, and supercritical, of the oscillating instabilities are observed in the Reynolds number/angle of attack domain. At very low Reynolds numbers of the laminar mode the instabilities are viscous effect dominated. The relation ships: $S t_{d} \cdot R e_{d} \approx$ and $\frac{f d^{2}}{v} \approx$ constant, hold either from experimental results or theoretical derivation. At large Reynolds numbers where the inertial effect 
dominated, the relationships hols: $S t_{d} \propto$ constant and $\frac{f d^{2}}{v} \propto R e_{d}$. In the regime between the limiting cases, the Strouhal number increases nonlinearly with Reynolds number and decreases abruptly as the shed vortices change from laminar to subcritical mode. The abrupt decrease of the shedding frequency due to the sudden increase of turbulence intensity as the characteristic mode changes from "laminar" to "subcritical" is justified by the experiments in free stream with high turbulence intensity.

\section{NOTATION}

C chord length of wing, $6 \mathrm{~cm}$

$d$ length of wing-section projection on crossstream plane

$f \quad$ frequency of instabilities in wake region $(\mathrm{Hz})$

$\ell \quad$ characteristic length scale in $x$ direction

$R e_{c} \quad$ Reynolds number based on chord length of wing $\left(=u_{w} C / v\right)$

$R e_{d}$ Reynolds number based on cross-stream projection of wing section $\left(=u_{w} d / v\right)$

$S t_{d} \quad$ Strouhal number of vortex shedding $\left(=f_{d} / u_{w}\right)$

$T$ characteristic time scale

$u \quad x$-component of local velocity

$u_{w} \quad$ free stream velocity

$v \quad y$-component of local velocity

$V \quad$ characteristic velocity scale in $y$ direction

$x \quad$ streamwise coordinate, originated from leading edge of wing on root-plane

$y$ spanwise coordinate, originated from leading edge of wing model on root-plane

$z \quad$ cross stream coordinate, originated from leading edge of wing model on root-plane

$\begin{array}{ll}\alpha & \text { root angle of attack } \\ \delta & \text { characteristic length scale in } y \text { direction }\end{array}$

$v \quad$ kinetic viscosity of air stream

\section{REFERENCES}

1. Lissaman, P.B.S., "Low Reynolds Number Airfoils," Annual Review of Fluid Mechanics, Vol. 15, pp. 223239 (1984).

2. Gad-el-Hak, M., "Control of Low-Speed Airfoil Aerodynamics," AIAA Journal, Vol. 28, No. 9, pp. 1537 1552 (1990).

3. Parker, R., "Resonance Effects in Wake Shedding from Parallel Plates: Some Experimental Observations," Journal of Sound and Vibration, Vol. 4, pp. 6272 (1966).

4. Blevins, R.D., Flow-Induced Vibration, 2nd ed., Van Nostrand Reinhold, New York, pp. 43-104 (1990).

5. Bollay, W. and Brown, C.D., "Some Experimental
Results on Wing Flutter," Journal of the Aeronautical Sciences, Vol. 8, pp. 313-318 (1941).

6. Marris, A.W., "A Review on Vortex Streets, Periodic Wakes, and Induced Vibration Phenomena," Journal of Basic Engineering, Vol. 86, pp. 185-194 (1964).

7. Berger, E. and Wille, R., "Periodic Flow Phenomena," Annual Review of Fluid Mechanics, Vol. 4, pp. 313340 (1972).

8. King, R., "A Review of Vortex Shedding Research and Its Application," Ocean Engineering, Vol. 4, pp. 141-171 (1977).

9. Griffin O.M., "Universal Similarity in the Wakes of Stationary and Vibrating Bluff Structures," Journal of Fluids Engineering, Vol. 103, pp. 52-58 (1981).

10. Levi, E., “A Universal Strouhal Law,” ASCE Journal of Engineering, Vol. 109, pp. $718-727$ (1983).

11. Griffin O.M., "Vortex Shedding from Bluff Bodies in a Shear Flow: a review," Journal of Fluids Engineering, Vol. 107, pp. 298-306 (1985).

12. Ho, C.-M. and Huerre, P., "Perturbed Free Shear Layers," Annual Review of Fluid Mechanics, Vol. 16, pp. 465-424 (1984).

13. Huerre, P. and Monkewita, P. A., "Local and Global Instabilities in Spatially Developing Flows," Annual Review of Fluid Mechanics, Vol. 22, pp. 473-537 (1990).

14. Motallebi, F. and Norbury, J.F., "The Effect of Base Bleed on Vortex Shedding and Base Pressure in Compressible Flow," Journal of Fluid Mechanics, Vol. 110, pp. 273-292 (1981).

15. Vassilopoulos, K., Gai, S.L., and Petrusma, M.S., "Unsteady Flow behind a Flunt Trailing Edge Aerofoil," AIAA Paper 95-0531, AIAA, Washington D. C. (1995).

16. Brooks, T. F. and Schlinker, R. H., "Progress in Rotor Broadband Noise Research," Vertica, Vol. 7, pp. 287 307 (1983).

17. Roshko, A., "On the Wake and Drag of Bluff Bodies," Journal of the Aerospace Science, Vol. 22, pp. 124135 (1955).

18. Simmons, J.E.L., "Similarities Between Two-Dimensional and Axisymmetric Vortex Wakes," Aero. Quarterly, Vol. 26, pp. 15-20 (1977).

19. Stuber, K. and Gharib, M., "Experiment on the Forced Wake of an Airfoil Transition from Order to Chaos," AIAA Paper 88-3840-CP, AIAA, Washington D. C. (1988).

20. Zaman, K.B.M.Q., McKinzie, D.J., and Rumsey, C.L., "A Natural Low-Frequency Oscillation of the Flow Over an Airfoil Near Stalling Conditions," Journal of Fluid Mechanics, Vol. 202, pp. 403-442 (1989).

21. Huang, R.F. and Lin, C.L., "Vortex Shedding and Shear-Layer Instability of Wing at Low-Reynolds Numbers," AIAA Journal, Vol. 33, pp. 1398-1430 
(1995).

22. Oertel, H., Jr., "Wakes Behind Blunt Bodies," Annual Reviews of Fluid Mechanics, Vol. 22, pp. 539-564 (1990).

23. Nishioka, M. and Sato, H., "Mechanism of Determination of the Shedding Frequency of Vortices Behind a Cylinder at Low Reynolds Numbers," Journal of Fluid Mechanics, Vol. 89, pp. 49-60 (1978).

24. Abbott, I.H. and von Doenhoff, A.E., Theory of Wing Section, Dover Publications, New York, N. Y., pp. 5053 (1959).

25. Gukhman, A.A., Introduction to the Theory of Similarity, Academic Press, New York, N. Y., pp. 19-47 (1965).

\section{NACA 0012機翼後方尾流區之頻 率特性}

\author{
李漢文黄榮 芳
}

國立堂澇科技大學機械工程系及研究所

摘 要

本文討論壹NACA 0012 機翼後方尾流區 中, 渦旋流逸之模態與頻率特性。由實驗結果獲悉 四種非稳態結構的模態: 層流、次臨界、過渡、與 超臨界, 自由流的紊流強度對於這些基本模態的範 图有很明顯的影響。由䔈驗結果與解析推導得知 : 在秥滞力主導區 (滑旋流逸通常為極低雷諾數的層 流模態), 史卓数與雷諾数成反比; 在慣性力主導 區（浮旋流逸通常為超臨界模態）, 史卓數维持常 数; 在秥滞力主導區與慣性力主導區之間, 史卓数 隨雷諾數非線性的增加, 但在渦旋流逸由層流模態 䡜變為次臨界模態時, 突然降到一個低值。自由流 的紊流強度對於高雷諾數的渦旋流逸影響較小。 